\title{
Evidências de validade de intervenções em grupo para orientação parental: Uma revisão integrativa
}

\author{
Evidence of validity of group interventions for parental guidance: An integrative review \\ Evidencia de validez de las intervenciones grupales para la orientación de los padres: Una revisión \\ integradora
}

Recebido: 11/04/2021 | Revisado: 18/04/2021 | Aceito: 22/04/2021 | Publicado: 08/05/2021

\author{
Marcli Firpo Bittencourt \\ ORCID: https://orcid.org/0000-0002-3931-2824 \\ Universidade Franciscana, Brasil \\ Email:marclifirpo@gmail.com \\ Pâmela Schultz Danzmann \\ ORCID: https://orcid.org/0000-0002-1438-4856 \\ Universidade Franciscana, Brasil \\ E-mail: pamelapsicologia10@gmail.com \\ Jessica Aguiar \\ ORCID: https://orcid.org/0000-0002-6390-7684 \\ Universidade Franciscana, Brasil \\ E-mail: aguiarjessica1@gmail.com \\ Marcia Pestana Santos \\ ORCID: https://orcid.org/0000-0002-4093-0291 \\ Universidade de Coimbra, Portugal \\ Email:marciansantos@gmail.com \\ Cristina Saling Kruel \\ ORCID: https://orcid.org/0000-0003-1996-7708 \\ Universidade Franciscana, Brasil \\ E-mail: cristinakruel@gmail.com.br \\ Simone dos Santos Paludo \\ ORCID: http://orcid.org/0000-0002-3281-5824 \\ Universidade Federal do Rio Grande, Brasil \\ E-mail: simonepaludo@yahoo.com.br \\ Josiane Lieberknecht Wathier Abaid \\ ORCID: https://orcid.org/0000-0002-5746-5349 \\ Universidade Franciscana, Brasil \\ E-mail: josianelwathier@gmail.com
}

\begin{abstract}
Resumo
O contexto familiar influencia significativamente o processo de desenvolvimento infantil. O modo como as famílias vivenciam as etapas evolutivas da vida influencia na maneira de enfrentamento às diversidades que intervêm nas relações parentais. Assim, os estudos voltados às práticas parentais e os meios de aprendizagem para melhor entender as relações possuem considerável relevância social. O presente artigo objetivou encontrar evidências de validade de intervenções em grupos voltados à orientação parental de pais. Foi realizada uma revisão integrativa composta por 17 artigos das bases de dados Biblioteca Virtual em Saúde (BVS), Literatura Latino-Americana e do Caribe em Ciências da Saúde (LILACS) e Scientific Eletroniv Library online (SciELO). Os resultados apontaram que a orientação parental realizada em grupos de pais gerou benefícios às famílias, considerando que esta prática favorece oportunidades de aprendizagem, apoio emocional a seus membros, ressignificação das formas de comunicação utilizadas nas interações pais e filhos e um conhecimento mais aprimorado por parte dos pais sobre os meios utilizados por eles na prevenção e enfrentamento a comportamentos disfuncionais e como isso interfere no bem-estar das crianças. Contudo, apesar dos diversos benefícios dos grupos de orientação parental, os artigos apresentam grandes diferenças metodológicas. Assim, conclui-se que ainda são necessários mais estudos interventivos sobre grupos parentais e as técnicas de treinamento de pais de crianças pequenas nos âmbitos familiares, escolares e outros contextos que a família esteja inserida.
\end{abstract}

Palavras-chave: Educação parental; Grupos parentais; Interação pais -filhos.

\begin{abstract}
The family context significantly influences the child development process. The way in which families experience the evolutionary stages of life, influence the way of coping with the diversity that intervenes in parental relationships. Thus, studies focused on parenting practices and the means of learning to better understand relationships, have
\end{abstract}


considerable social relevance. This article aimed to find evidence of validity of interventions in groups aimed at parental guidance of parents and / or caregivers of children. An integrative review comprising 17 articles from the VHL, LILACS and SciELO databases was carried out. The results showed that the parental guidance carried out in groups of parents generated brought benefits to the families, considering that this practice favors learning opportunities, emotional support to its members, reframing the forms of communication used in the interactions between parents and children, and a better knowledge on the part parents about the means used by them to prevent and cope with dysfunctional behaviors and how it interferes with the children's well-being. However, despite the diverse benefits of parental guidance groups, the articles feature bring great methodological differences. Thus, it is concluded that more interventional studies on parental groups and the training techniques of parents in the age group proposed in the family, school and other contexts in that the family is inserted are still necessary.

Keywords: Parental education; Parental groups; Parent-child interaction.

\section{Resumen}

El contexto familiar influye significativamente en el proceso de desarrollo infantil. La forma en que las familias experimentan las etapas evolutivas de la vida, influye en la forma de afrontar la diversidad que interviene en las relaciones parentales. Por tanto, los estudios centrados en las prácticas parentales y los medios para aprender a comprender mejor las relaciones tienen una relevancia social considerable. El presente artículo tuvo como objetivo encontrar evidencia de la validez de las intervenciones en grupos dirigidos a la orientación parental de los padres de niños pequeños. Se realizó una revisión integradora de 17 artículos de las bases de datos Biblioteca Virtual de Salud ( BVS), Literatura Latino americana y del Caribe en Ciencias de la Salud (LILACS) y Biblioteca Científica Eletroniv en línea (SciELO) . Los resultados mostraron que la realizada en grupos de padres trajo beneficios a las familias, considerando que esta práctica favorece las oportunidades de aprendizaje, el apoyo emocional a sus miembros, redefiniendo las formas de comunicación utilizadas en las interacciones entre padres e hijos, y un mejor conocimiento. por parte de los padres sobre los medios que utilizan para prevenir y hacer frente a los comportamientos disfuncionales y cómo interfieren en el bienestar de los niños. Sin embargo, a pesar de los diversos beneficios de los grupos de orientación parental los artículos traen grandes diferencias metodológicas. Así, se concluye que aún son necesarios más estudios intervencionistas sobre grupos parentales y las técnicas de formación de los padres en el grupo de edad propuesto en la familia, la escuela y otros contextos en los que se inserta la familia.

Palabras clave: Educación de los padres; Grupos de padres; Interacción entre padres e hijos.

\section{Introdução}

A família é um grupo natural que, com o passar do tempo, foi se reestruturando conforme as modificações culturais da sociedade, elaborando assim suas relações de interação. As mudanças entre as relações familiares ao longo dos anos, fruto das transformações que a família sofre, apontam para inúmeros problemas em relação às práticas comportamentais das crianças. De acordo com Lubi (2003), os pais, enquanto formadores do núcleo familiar, são, indiscutivelmente, poderosa fonte de influência no desenvolvimento da criança, o que lhes atribui grande responsabilidade.

A habilidade dos pais em conhecer e auxiliar no entendimento das necessidades de suas crianças, orientando e apoiando, tanto nos ganhos quanto nas perdas, e proporcionando emoções positivas, pode revelar o grau do desenvolvimento de confiança e boa vontade para relacionar-se com os outros através de meios positivos. Atitudes arbitrárias e inconsistentes de falta de afeto e cobrança exacerbada denunciam, provavelmente, o surgimento de problemas que, uma vez emergidos, alimentarão a frustração, o medo, a agressão ou altos níveis de ansiedade nas crianças. Gomide (2001) explica que as diferentes maneiras utilizadas pelos pais no cuidado de seus filhos podem estar correlacionadas tanto com o desenvolvimento saudável da criança como podem vincular-se ao desenvolvimento de comportamentos antissociais.

Conforme explicam Caballo e Simon (2004), a família proporciona o primeiro e mais importante contexto social, emocional, interpessoal, econômico e cultural para o desenvolvimento humano. Como resultado, as relações familiares têm uma profunda influência sobre o bem-estar das crianças e de todos aqueles que compõem o núcleo familiar. Pensando nessa problemática, busca-se meios de orientar as relações parentais por meio da técnica de treinamento de pais embasada na abordagem cognitivo-comportamental, cujo objetivo principal consiste em identificar padrões de comportamento, mudando a ênfase da família dos comportamentos problemáticos, indicando, a partir disso, ferramentas para alterar essas percepções de forma positiva. 
O objetivo da abordagem cognitivo-comportamental familiar consiste em promover a reestruturação cognitiva essencialmente por meio de intervenções e técnicas cognitivas e comportamentais. A modificação cognitiva leva a alterações nos sistemas afetivos e comportamentais que se encontravam anteriormente desadaptados. Essa técnica busca, através de grupos de treinamento de pais, orientar os mesmos para práticas parentais positivas (Peterson, 2011).

A abordagem cognitiva baseia-se na teoria da aprendizagem social a qual, entre outras, parte do pressuposto de que o ambiente, as características temperamentais e o comportamento de uma pessoa determinam-se reciprocamente e que o comportamento é um fenômeno dinâmico, em evolução, pois os contextos influenciam o comportamento;e o comportamento, por sua vez, molda os contextos (Asbahr \& Ito, 2008).

Os pais costumam buscar tratamento para seus filhos frequentemente para pedir orientação sobre como podem ajudálos a superarem seus problemas ou medos. Famílias com pensamentos e crenças adaptativas e que estimulam o filho a enfrentar as situações difíceis de uma forma positiva e funcional ajudam muito na redução dos sintomas de ansiedade deste, pois são modelos de comportamento importantes na sua vida. Para McMahon (2007), os problemas na relação pais-filhos têm impacto na apresentação e manutenção do comportamento desadaptativo da criança, sendo o envolvimento dos pais nesta problemática uma prática positiva.

O contexto interpessoal e social da criança, incluindo pares e familiares, também é importante de se considerar no desenvolvimento das crianças e deve ser levado em consideração ao se analisar a realidade emocional dos mesmos. Esses resultados evidenciaram a necessidade da criação de estratégias que possibilitem ao sujeito generalizar suas conquistas obtidas no contexto clínico para o ambiente natural; assim, os ganhos adquiridos na psicoterapia seriam aplicados nos locais em que o problema de comportamento se apresentava (Caminha et al., 2011). Ademais, o treinamento de pais pode ser conceituado no contexto clínico e da saúde como um enfoque para o tratamento dos problemas de comportamento que utiliza estratégias por meio das quais os pais são treinados a modificar a interação pais-filhos (Mcmahon, 2007).

A finalidade desta estratégia consiste em estimular o comportamento pró-social e diminuir ou eliminar os comportamentos desadaptativos. Assim, este estudo objetivou identificar na literatura evidências de prevenção e promoção de práticas educativas parentais com pais de crianças a partir de grupos de educativos.

\section{Metodologia}

Optou-se por uma revisão integrativa de literatura, pois segundo Mendes, Silveira e Galvão (2008) este é um método de pesquisa que permite a busca, a avaliação crítica e a síntese das evidências disponíveis quanto ao tema estudado, de forma a ter como produto final o estado atual do conhecimento investigado e a identificação de lacunas que direcionam para o desenvolvimento de pesquisas futuras. Para Sousa, Silva e Carvalho (2010), a revisão integrativa emerge como uma metodologia que proporciona a síntese do conhecimento e a incorporação da aplicabilidade de resultados de estudos significativos na prática.

A construção da revisão integrativa procedeu-se em seis etapas: 1) identificação do tema; 2) formulação da pergunta norteadora; 3) delimitação dos descritores e seleção das bases de dados; 4) estabelecimento de critérios de exclusão e inclusão dos trabalhos; 5) leitura dos artigos selecionados e interpretação dos resultados; 6) leitura dos artigos selecionados e interpretação dos resultados. A questão norteadora deste estudo foi: quais são as evidências de mudanças comportamentais de pais e crianças, a partir de grupos de orientação parental? Ainda, os artigos foram classificados quanto ao nível de evidência dos dados (Stillwell et al., 2010). Essa classificação é um sistema que leva em consideração a qualidade das evidências sendo classificada em sete níveis. 
No nível 1, as evidências são provenientes de revisão sistemática ou meta-análise de todos relevantes ensaios clínicos randomizados controlados ou oriundos de diretrizes clínicas baseadas em revisões sistemáticas de ensaios clínicos randomizados controlados.

No nível 2, as evidências são derivadas de pelo menos um ensaio clínico randomizado controlado bem delineado.

No nível 3, as evidências são obtidas de ensaios clínicos bem delineados sem randomização.

No nível 4, as evidências são provenientes de estudos de coorte e de casos-controle bem delineados.

No nível 5, as evidências são originárias de revisão sistemática de estudos descritivos e qualitativos.

No nível 6, as evidências são derivadas de um único estudo descritivo ou qualitativo.

E no nível 7, as evidências são oriundas de opinião de autoridades e/ou relatório de comitês de especialistas.

A questão de pesquisa foi elaborada por meio da estratégia PICO (paciente, intervenção, comparação e resultados), em que: $\mathrm{P}=$ pais/cuidadores de crianças; $\mathrm{I}=$ =Intervenção com Orientação Parental; $\mathrm{C}$ =modelos de intervenção; $\mathrm{O}=$ percepção sobre mudanças no comportamento de pais e crianças. Partindo da primeira etapa, buscou-se compreender se a orientação parental realizada em grupos trouxe benefícios às famílias.

Estabeleceu-se como critérios de inclusão: artigos publicados na íntegra, disponíveis eletronicamente, nas línguas portuguesa, inglesa e/ou espanhola, cujos resultados adviessem de intervenções relacionadas com a educação parental e o treinamento de pais. E como critérios de exclusão: artigos repetidos em mais de uma base ou que não focaram o assunto de forma contextualizada como na pergunta norteadora, bem como os indisponíveis online, e artigos que tratassem de revisões de literatura e materiais como dissertações e teses.

A revisão ocorreu por meio das plataformas digitais Biblioteca Virtual em Saúde (BVS), Literatura da América Latina e do Caribe (Lilacs) e Scientific Electronic Library Online SciELO, no período de junho de 2019 a dezembro de 2020. Desse modo, utilizaram-se os seguintes Descritores das Ciências da Saúde: Educação Parental - AND, treinamento de pais - OR e treinamento de pais AND relação pais e filhos, na base de dados LILACS; Educação Parental AND treinamento de pais, OR treinamento de pais AND relação pais e filhos, na base de dados SCIELO; por fim, Education, Non professional AND parentchild relations, OR Education, Non Professional and parenting, na base de dados LILACS, sendo realizada a pesquisa como cruzamento simultâneo dos descritores, utilizando os booleanos AND(e) e OR (OU) (Tabela 1).

Tabela 1. Bases de Dados e Respectivos Descritores Utilizados na Pesquisa.

\begin{tabular}{ll}
\hline BASE DE DADOS & DESCRITORES CONTROLADOS \\
\hline LILACS & $\begin{array}{l}\text { Educação parental and Treinamento de pais } \\
\text { Treinamento de pais and relação pais-filhos }\end{array}$ \\
& $\begin{array}{l}\text { Educação parental and treinamento de pais } \\
\text { Treinamento de pais and relação pais-filhos }\end{array}$ \\
SciELO & $\begin{array}{l}\text { Education, Non professional and parent-child } \\
\text { relations } \\
\text { Education, Nonprofessional and parenting }\end{array}$ \\
\hline
\end{tabular}

Fonte: Autores.

Após a seleção final dos artigos (Figura 1), realizou-se uma análise de conteúdo temática (Bardin, 2011). As três categorias extraídas foram: Práticas e estilos parentais; Temáticas, abordagens e contextos que podem ser utilizadas nas orientações; e Principais evidências de benefícios da orientação parental. Os resultados são apresentados a seguir. 


\section{Resultados}

Foi composto por 17 artigos (Figura 1).

Figura 1- Fluxograma de seleção e Identificação dos Artigos.
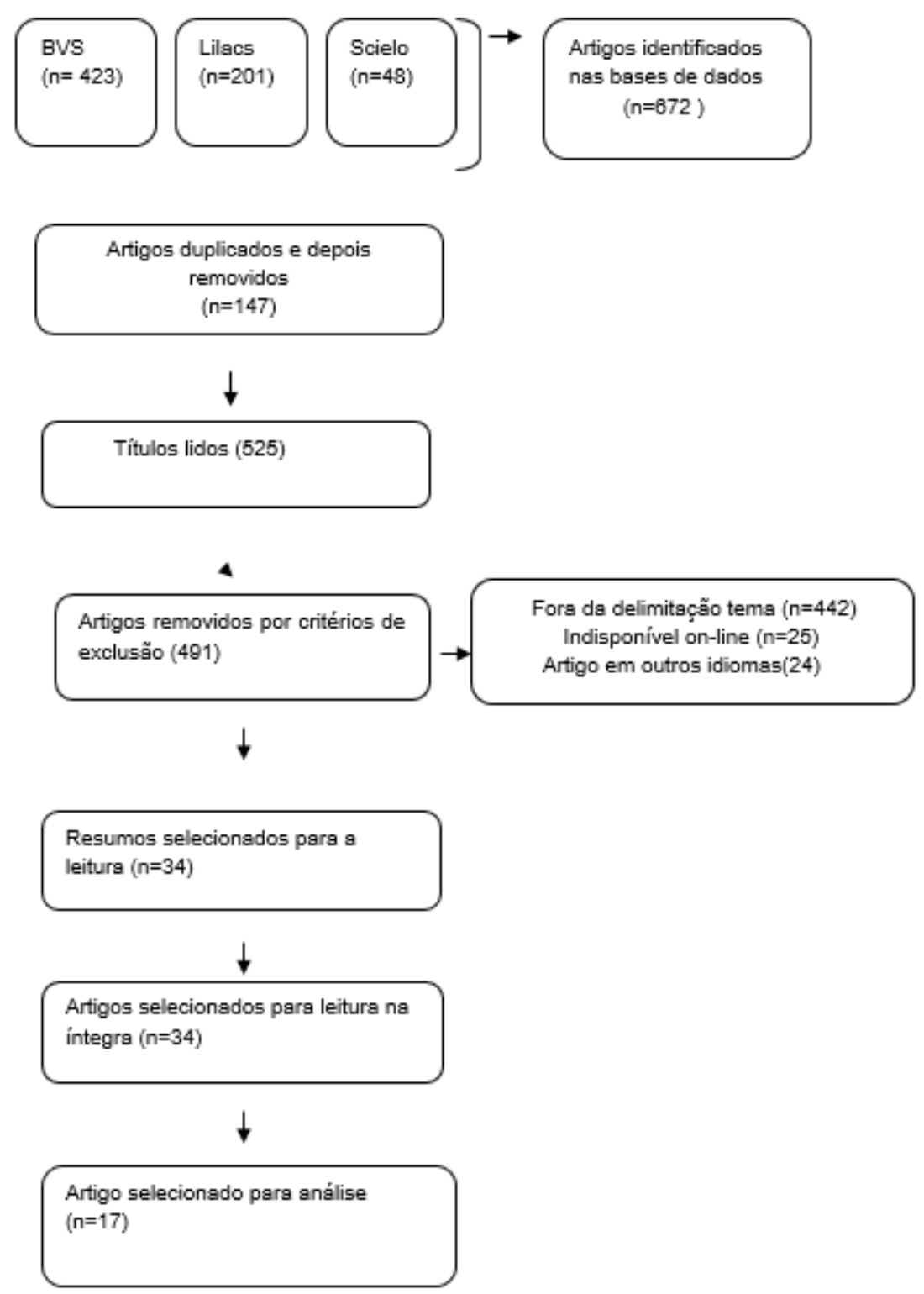

Fonte: Autores.

Assim, apresentam-se os 17 artigos selecionados na Tabela 2. 
Tabela 2. Identificação dos Artigos Selecionados Quanto aos Participantes, Objetivos, Tipo de Estudo, Nível de Evidência e Resultados.

\begin{tabular}{|c|c|c|c|c|c|}
\hline $\begin{array}{l}\text { AUTORES/ } \\
\text { Bases de } \\
\text { dados }\end{array}$ & $\begin{array}{c}\text { TÍTULO } \\
\text { LOCAL DATA DE } \\
\text { PUBLICAÇÃO }\end{array}$ & PARTICIPANTES & OBJETIVOS & $\begin{array}{c}\text { TIPO DE } \\
\text { ESTUDO/NÍVEL DE } \\
\text { EVIDÊNCIA* }\end{array}$ & RESULTADOS \\
\hline
\end{tabular}

$\begin{array}{lll} & \text { Orientação de pais: } & \begin{array}{l}\text { Grupos de mães e } \\ \text { de orientação }\end{array} \\ \text { 1. Mendes e } & \text { partilhar } & \begin{array}{l}\text { individual a pais, de } \\ \text { classe média }\end{array} \\ \text { Biasoli-Alves } & \begin{array}{l}\text { conhecimentos sobre } \\ \text { desenvolvimento e }\end{array} & \begin{array}{l}\text { popular, atendidos } \\ \text { (2005) }\end{array} \\ \text { práticas de educação } & \text { segundo projetos de } \\ \text { como estratégia de } & \text { extensão de } \\ & \text { intervenção. } & \text { serviços à } \\ & & \text { comunidade. }\end{array}$

Discutir uma proposta de orientação para famílias desenvolvido com grupos de mães, e de orientação individual a pais, atendidos segundo projetos de extensão de serviços à comunidade.
Os resultados permitiram analisar mudança nas percepções dos pais sobre o comportamento e atitudes dos filhos. Houve evolução na forma dos mesmos observarem seu cotidiano e suas relações com as práticas de educação, levando a um maior envolvimento nas atividades

realizadas pelos filhos,
Artigo de pesquisa,

Transversal e

Longitudinal, Nível de evidência VI.
O programa atingiu seus objetivos, ressaltando que trabalhos de prevenção e intervenção com os pais são necessários para aumento da qualidade na interação familiar.

$\begin{array}{ll}\text { 2. Weber, } & \text { Qualidade na } \\ \text { Brandenburg e } & \text { Interação Familiar } \\ \text { Salvador } & \text { (PQIF): } \\ \text { (2006) } & \text { Orientação e } \\ \text { LILACS } & \begin{array}{l}\text { treinamento para } \\ \text { pais. }\end{array} \\ \end{array}$

93 Tornar os pais conscientes participantes. de seus comportamentos e das implicações.
Artigo de pesquisa evidência VI.

$\begin{array}{lll}\text { 3. Pardo et al. } & \begin{array}{l}\text { Entrevista Inicial } \\ \text { como Suporte para }\end{array} & \begin{array}{l}\text { Mães participantes } \\ \text { de grupos de } \\ \text { orientação }\end{array} \\ \text { BVS } & \text { Intervenções em } & \text { realizados em uma } \\ \text { Grupos de } & \text { Clínica escola; }\end{array}$

\section{Apresentar os resultados} obtidos nas entrevistas iniciais com mães que participaram de grupos no período de 2010 e 2013
Artigo de pesquisa, Transversal, Nível de evidência VI.
Os resultados indicaram a necessidade de trabalhar a percepção da influência recíproca entre os comportamentos da mãe e da criança e refletir sobre a melhor forma de ajustá-los com o intuito de potencializar o desenvolvimento infantil.

Notou-se que ambos os grupos obtiveram resultados positivos; no entanto, G1 destacou-se no aumento das habilidades sociais dos pais e das crianças e G2, na diminuição dos

26 pais/cuidadores,
os quais se inscreveram em um procedimentos de intervenção programa de intervenção com

Silva, Laura

Treinamento de habilidades sociais Moreira Borelli (2012) LILACS educativas parentais: comparação de procedimentos a partir do tempo de intervenção. pais no Centro de Psicologia Aplicada de uma

Universidade no interior paulista, em uma clínica-escola. com pais que buscaram Artigo de pesquisa, promover melhores interações Transversal e

sociais com seus filhos, por Longitudinal, Nível de meio do treinamento e fortalecimento das habilidades sociais. evidência VI. problemas de comportamento. Conclui-se que a intervenção de maior duração pode proporcionar a maior aquisição e consolidação de habilidades e aponta-se para a necessidade da realização de trabalhos preventivos com a população e de estudos comparativos para o aprimoramento de procedimentos de intervenção.

\begin{tabular}{|c|c|c|c|c|c|}
\hline $\begin{array}{l}\text { 5. Solís- } \\
\text { Câmara, } \\
\text { Medina e Diaz } \\
(2015) \\
\text { SciELO }\end{array}$ & $\begin{array}{l}\text { Análisis compara- } \\
\text { tivo de predictores } \\
\text { potenciales de } \\
\text { prácticas disciplina- } \\
\text { rias severas con } \\
\text { preescolares, antes y } \\
\text { después de un } \\
\text { entrenamiento para } \\
\text { padres. }\end{array}$ & $\begin{array}{l}\text { Grupos compostos } \\
\text { por } 60 \text { pais ( } 41 \\
\text { mães e } 19 \text { pais) de } \\
\text { crianças de } 3 \text { a } 5 \\
\text { anos. }\end{array}$ & $\begin{array}{l}\text { Determinar, através de uma } \\
\text { revisão sistemática, se um } \\
\text { treinamento em pautas de } \\
\text { criação dirigido a pais com } \\
\text { crianças de três a cinco anos } \\
\text { de idade modificaria as } \\
\text { práticas disciplinares severas, } \\
\text { bem como alguns preditores } \\
\text { potenciais dessas práticas, } \\
\text { como, por exemplo a } \\
\text { coragem-agressão e o estresse } \\
\text { dos pais, da mesma forma que } \\
\text { o estresse na interação entre } \\
\text { pais e filhos. }\end{array}$ & $\begin{array}{l}\text { Artigo de pesquisa, } \\
\text { Estudo longitudinal } \\
\text { /Nível de evidência VI. }\end{array}$ & $\begin{array}{l}\text { Os resultados discutem-se em } \\
\text { termos da importância da } \\
\text { modificação das práticas } \\
\text { disciplinares severas e seus } \\
\text { efeitos negativos nas crianças, } \\
\text { bem como do uso adequado } \\
\text { de técnicas cognitivo } \\
\text { condutores para modificar } \\
\text { positivamente as relações } \\
\text { negativas entre os pais e } \\
\text { a criança. }\end{array}$ \\
\hline
\end{tabular}




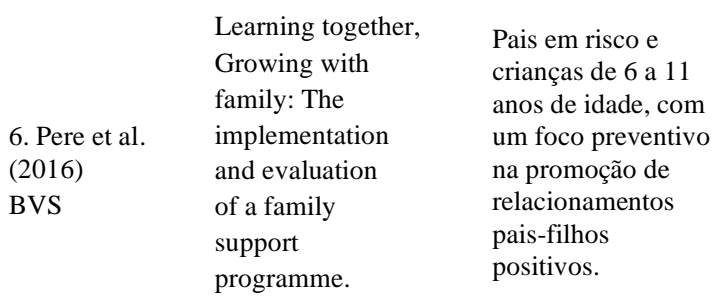

Learning together, Growing with family: The

6. Pere et al. implementation

(2016)

programme.
Examinar a qualidade da implementação do programa e sua influência nos resultados do em uma amostra de 425 pais e 138 facilitadores extraídos do primeiro ensaio.
Análise comparativa, estudo longitudinal, evidência III
Os resultados mostraram alta qualidade de implementação no que diz respeito ao facilitador do grupo e aos fatores de organização do programa, seguida pela coordenação com os serviços e facilidades de apoio oferecidas aos participantes e, por fim, pelos fatores de fidelidade $\mathrm{e}$ etapas anteriores da organização. Os resultados dos grupos focais confirmaram que as etapas anteriores foram desafiadoras e ofereceram as estratégias mais eficazes na implantação do programa em outro contexto.
Os bebês que receberam a intervenção ficaram em conformidade com os comandos maternos e apresentaram níveis mais mães de bebês de 12 a 15 meses recrutadas em clínica pediátrica de um grande hospital de origem hispânica.
Comparar dois grupos de bebês, um que recebeu intervenção e outro que não recebeu após um determinado período de tempo.
Análise comparativa, estudo longitudinal, evidência III. baixos de problemas de externalização e internalização de comportamento; as mães que receberam a intervenção apresentaram também comportamentos positivos, $\mathrm{o}$
Opportunity for High-Risk Families.
Estudo transversal

8. Boing e

Crepaldi

(2016)

SciELO
Relação pais e filhos: compreendendo o interjogo das relações parentais e coparentais. e famílias biparentais com crianças de 5 a 7 anos de idade com desenvolvimento normativo.
Compreender os estilos e práticas parentais e seus efeitos no desenvolvimento das crianças, relacionando estilos parentais e coparentalidade. que sugere ser essa prática positiva para a orientação de pais.

O conjunto dos resultados permitiu o delineamento das relações entre a coparentalidade e estilos parentais e poderão subsidiar intervenções psicológicas e psicoeducativas promotoras do desenvolvimento das crianças e suas famílias, em diferentes contextos.

Transversal, Nível evidência VI.
Os resultados dos grupos focais confirmaram que as intervenções parentais positivas favorecem a saúde mental dos filhos, foram desafiadoras e ofereceram as estratégias mais eficazes.
Parental Systems: A

et al.

(2016)

Parental Engagement in Programmes for

BVS Child Mental Health-

Connect, Attend,

Participate, Enact (CAPE)
Avaliar as condições em que programa foi implementado

425 pais e 138 para contribuir com seu desenvolvimento e aprimoramento em ensaios futuros de interação parental.
Artigo de pesquisa, Transversal, Nível de evidência VI.

\begin{tabular}{lll}
\hline & & \\
10. Garcia e & Entrenamiento & \\
Grau & Conductual y & Cinco mães, dois \\
$(2017)$ & Atención Plena sobre & pais e seis filhos. \\
LILACS & el Estrés Parental y & \\
& Relación Padre-Hijo. &
\end{tabular}

Avaliar a efetividade de um tradicional procedimento de treinamento de pais, combinado a um treinamento de mindfulness na redução do estresse parental e no aumento de interações positivas entre país e filhos.
Artigo de pesquisa,

Transversal e

longitudinal, Nível de evidência VI.

Os resultados demonstraram que o treinamento combinado foi efetivo na redução do estresse parental, no aumento de mindfulness e na melhora da relação entre país e filhos.

\begin{tabular}{|c|c|c|c|c|c|}
\hline $\begin{array}{l}\text { 11. Coelho e } \\
\text { Murta (2017) } \\
\text { LILACS }\end{array}$ & $\begin{array}{l}\text { Treinamento de pais } \\
\text { em grupo: um relato } \\
\text { de experiência. }\end{array}$ & $\begin{array}{l}\text { Cinco mães e dois } \\
\text { pais participaram de } \\
\text { uma intervenção } \\
\text { grupal, com } 20 \\
\text { sessões de } 90 \\
\text { minutos e } \\
\text { periodicidade } \\
\text { semanal. }\end{array}$ & $\begin{array}{l}\text { Ensinar os pais a observar e } \\
\text { descrever o comportamento } \\
\text { da criança e a serem agentes } \\
\text { mais eficazes de reforço, } \\
\text { aumentando a frequência, a } \\
\text { variedade e a extensão de } \\
\text { reforços sociais para as } \\
\text { crianças e reduzindo a }\end{array}$ & $\begin{array}{l}\text { Artigo de pesquisa, } \\
\text { Transversal, Nível de } \\
\text { evidência VI. }\end{array}$ & $\begin{array}{l}\text { Os resultados, avaliados por } \\
\text { entrevistas e checklist, } \\
\text { indicaram aumento nas práticas } \\
\text { educativas parentais positivas, } \\
\text { desenvolvimento de } \\
\text { habilidades sociais educativas } \\
\text { e desenvolvimento de } \\
\text { estratégias saudáveis de }\end{array}$ \\
\hline
\end{tabular}


frequência de

comportamentos verbais

competidores, como críticas,

ordens e questionamentos. enfrentamento a estressores externos nos participantes.

$\begin{array}{ll}\text { 12. Oliveira, } & \text { Manejo dos pais } \\ \text { Gurtal e Reis } & \begin{array}{l}\text { frente à expressão de } \\ \text { (2018) }\end{array} \\ \text { rCIELO } & \text { raiva dos filhos. }\end{array}$

SCIELO raiva dos filhos.
Estudo transversal /

26 pais de crianças

em idade escolar

com

desenvolvimento

normativo.
Investigar o manejo dos pais frente à expressão da emoção raiva dos filhos dos sexos masculino e feminino.
Artigo de pesquisa,

Transversal, Nível de evidência VI.
Os resultados indicaram a necessidade de trabalhar a percepção da influência recíproca entre os comportamentos da mãe e da criança e refletir sobre a melhor forma de ajustá-los com o intuito de potencializar o desenvolvimento infantil e minimizar o stress parental

$\begin{array}{ll} & \text { Programa de } \\ \text { 13. Neufeld et } & \text { Orientação de Pais } \\ \text { al. } & \text { em Grupo: Um } \\ \text { (2018) } & \text { estudo exploratório } \\ \text { LILACS } & \text { na abordagem } \\ & \text { Cognitivo } \\ & \text { Comportamental }\end{array}$

7 pais de crianças em idade escolar com desenvolvimento normativo.
Avaliar de forma preliminar os efeitos destas intervenções, baseando-se nos escores do Inventário de Estilos Parentais (IEP), identificando se houve diferenças entre pré e pósteste no que tange ao estilo parental.
Artigo de pesquisa, Transversal e

longitudinal, Nível de evidência VI.
Os pais, em geral, conseguiram melhorar seu estilo parental e apresentar diminuição nas práticas parentais negativas.

\begin{tabular}{|c|c|c|c|c|c|}
\hline $\begin{array}{l}\text { 14. Axford et } \\
\text { al. } \\
(2018) \\
\text { BVS }\end{array}$ & $\begin{array}{l}\text { The effectiveness of } \\
\text { the Inspiring Futures } \\
\text { parenting programme } \\
\text { in improving } \\
\text { behavioural and } \\
\text { emotional outcomes } \\
\text { in primary school } \\
\text { children with } \\
\text { behavioural or } \\
\text { emotional } \\
\text { difficulties: study } \\
\text { protocol for a } \\
\text { randomised } \\
\text { controlled trial. }\end{array}$ & $\begin{array}{l}\text { Grupo de pais de } \\
\text { crianças que } \\
\text { cursavam o ensino } \\
\text { fundamenta e que } \\
\text { foram divididos em } \\
\text { dois grupos, sendo } \\
\text { um grupo controle } \\
\text { por } 12 \text { semanas no } \\
\text { total de } 248 \\
\text { participantes no } \\
\text { Reino Unido. }\end{array}$ & $\begin{array}{l}\text { Determinar a eficácia do } \\
\text { programa Inspiring Futures no } \\
\text { resultado primário de } \\
\text { dificuldades comportamentais } \\
\text { e emocionais de crianças da } \\
\text { escola primária identificadas } \\
\text { como tendo dificuldades } \\
\text { comportamentais ou } \\
\text { emocionais. }\end{array}$ & $\begin{array}{l}\text { Artigo de pesquisa. } \\
\text { Ensaio Clínico } \\
\text { Randomizado, nível II. }\end{array}$ & $\begin{array}{l}\text { O intuito da pesquisa era } \\
\text { avaliar o Programa Parenting } \\
\text { Inspiring Futures do Reino } \\
\text { Unido, tendo uma resposta } \\
\text { positiva às intervenções nas } \\
\text { dúvidas quanto às práticas } \\
\text { parentais e desenvolvimento } \\
\text { infantil, sendo o programa } \\
\text { avaliado como um facilitador } \\
\text { na comunicação pais e filhos, } \\
\text { diante da realidade relatada de } \\
\text { dificuldades nas relações } \\
\text { parentais. }\end{array}$ \\
\hline
\end{tabular}

\begin{tabular}{|c|c|c|c|c|c|}
\hline $\begin{array}{l}\text { 15. Kazdin et } \\
\text { al. } \\
(2018) \\
\text { BVS }\end{array}$ & $\begin{array}{l}\text { Parent management } \\
\text { Training for } \\
\text { Conduct problems } \\
\text { In children: } \\
\text { enhancing } \\
\text { Treatment to } \\
\text { Improve } \\
\text { therapeutic change. }\end{array}$ & $\begin{array}{l}138 \text { famílias, } 39 \\
\text { meninas e } 99 \\
\text { meninos, com } \\
\text { idades entre } 6 \text { e } 13 \\
\text { anos }\end{array}$ & $\begin{array}{l}\text { Avaliar o impacto de vários } \\
\text { aprimoramentos para otimizar } \\
\text { fatores comuns e de placebo } \\
\text { para aumentar a mudança } \\
\text { terapêutica. }\end{array}$ & $\begin{array}{l}\text { Artigo de pesquisa, } \\
\text { Estudo transversal e } \\
\text { longitudinal / Nível de } \\
\text { evidência VI. }\end{array}$ & $\begin{array}{l}\text { Os resultados indicaram que as } \\
\text { crianças e os pais obtiveram } \\
\text { melhora acentuada em todas as } \\
\text { medidas de resultado do } \\
\text { tratamento, como diminuição } \\
\text { de stress dos pais e aumento de } \\
\text { vínculo com os filhos. }\end{array}$ \\
\hline $\begin{array}{l}\text { 16. Vásquez et } \\
\text { al. } \\
(2020) \\
\text { BVS }\end{array}$ & $\begin{array}{l}\text { Social Factors } \\
\text { Associated with the } \\
\text { Effectiveness of a } \\
\text { Spanish Parent } \\
\text { Training Program - } \\
\text { An Opportunity to } \\
\text { Reduce Health } \\
\text { Inequality Gap in } \\
\text { Families. }\end{array}$ & $\begin{array}{l}257 \text { pais., sendo } \\
87 \% \text { de mães } \\
\text { divididos em três } \\
\text { grupos, conforme } \\
\text { idade dos filhos de } \\
2 \text { a } 17 \text { anos }\end{array}$ & $\begin{array}{l}\text { Identificar as características } \\
\text { sociais dos pais que } \\
\text { determinam o sucesso do } \\
\text { programa PSP (Competências } \\
\text { Parentais para Famílias) em } \\
\text { relação ao suporte social, } \\
\text { habilidades parentais, estresse } \\
\text { parental e negativo } \\
\text { comportamentos entre } \\
\text { crianças. }\end{array}$ & $\begin{array}{l}\text { Artigo de pesquisa, } \\
\text { Transversal, Nível de } \\
\text { evidência VI. }\end{array}$ & $\begin{array}{l}\text { O estresse dos pais diminuiu } \\
\text { entre os pais de diferentes } \\
\text { níveis sociais, que estavam } \\
\text { desempregados e eram } \\
\text { homens. O apoio social } \\
\text { aumentou entre os pais mais } \\
\text { jovens, com dificuldades } \\
\text { socioeconômicas, favorecendo } \\
\text { as relações parentais. }\end{array}$ \\
\hline
\end{tabular}

$\begin{array}{lll} & & \\ & & \text { Results of the 3 } \\ \text { 17. Marsh et } & \begin{array}{l}\text { Pillars Study (3PS), a } \\ \text { relationship-based }\end{array} & \\ \text { al. } & \text { programme targeting } & \text { de } 2 \text { a } 4 \text { anos de } \\ (2020) & \text { parent-child } & \text { idade. } \\ \text { BVS } & \text { interactions, healthy } & \\ & \text { lifestyle. }\end{array}$

Apresentar um programa de estilo de vida saudável para pais de crianças em idade pré-escolar que promovem rotinas em torno de comportamentos de estilo de vida saudáveis, incluindo sono, uso limitado da tela e refeições em família, dentro do contexto positivo.
Identificar as característica determinam o sucesso do programa PSP (Competências relação ao suporte social, habilidades parentais, estresse comportamentos entre crianças.
$\mathrm{O}$ intuito da pesquisa era avaliar o Programa Parenting Unido, tendo uma resposta positiva às intervenções nas idas quanto às práticas infantil, sendo o programa avaliado como um facilitador na comunicação pais e filhos, dificuldades nas relações parentais.
Os resultados indicaram que as crianças e os pais obtiveram medidas de resultado do tratamento, como diminuição de stress dos pais e aumento de vínculo com os filhos. 
Quanto ao objetivo das publicações, os autores buscaram, em sua maioria, descrever as práticas parentais, o modo como o treinamento de pais interfere nas boas práticas, a relação dos castigos e punições no desenvolvimento infantil e o que acarreta na postura das crianças o papel da coparentalidade como fundamental no atendimento aos filhos. Neste contexto, as técnicas da abordagem cognitiva-comportamental foram utilizadas como facilitadoras da mudança de comportamento dos pais e auxiliaram no combate aos problemas de saúde mental que levam aos castigos dos filhos.

Após a análise dos artigos, foram encontradas as seguintes categorias: Práticas e estilos parentais; Temáticas, abordagens e contextos que podem ser utilizados na orientação de pais; e Principais evidências de benefícios da orientação parental. A seguir, descreve-se e exemplifica-se cada categoria. Posteriormente, descrevem-se os artigos analisados de acordo com as categorias encontradas.

\section{Resultados}

\subsection{Práticas e Estilos Parentais}

Categoria que aborda as descrições sobre práticas de cuidadores (punição, conversa) e também sobre seus estilos parentais. Nesta classe, estão incluídos dados relatados na literatura pesquisada sobre o que interfere nas práticas educativas. Ex.: quando se aplica uma punição em função do humor; como no trecho "humor instável - quando estava brava com coisas do emprego chegava em casa e descontava nos meus filhos, agora eu não tenho mais transferido os problemas de lá de fora para dentro da minha casa" (artigo n. 11)

Quanto às práticas educativas e estilos parentais evidenciados nos estudos, destaca-se a predominância do estilo parental de responsividade através da orientação dada aos pais nas atividades em grupo. A participação da figura paterna também foi ressaltada como primordial na transmissão de valores, limites e afeto, o que ocasionou diminuição do stress das mães na execução de seus papéis como educadoras e responsáveis diretamente pelo desenvolvimento dos filhos (Boing \& Crepaldi 2016; Garcia \& Grau, 2017; Mendes \& Biasoli-Alves, 2005; Pere et al., 2016; Piotrowska et al., 2016; Oliveira, Gurtal \& Reis, 2018; Sólis-Câmera, Medina \& Diaz, 2015; Vásquez et al., 2020).

Os estilos parentais permeiam as práticas educativas e se apresentam no modo com que os pais agem e se posicionam quanto ao desempenho dessas práticas. Para Marsh et al. (2020), identifica-se uma evolução após as intervenções grupais na forma de os pais observarem o que se passa no cotidiano de sua casa e na vida da criança e suas relações com as práticas de educação que eles vêm utilizando como forma de educar. O desenvolvimento da criança é afetado por diversos fatores e os pais precisam estar atentos a eles para poder atender as necessidades dos filhos e assim poder orientá-los da melhor maneira possível dentro de um ambiente de carinho e respeito (Garcia \& Grau, 2017; Oliveira, Gurtal \& Reis, 2018). A falta de exigência dos pais ou o detrimento de práticas disciplinares junto aos filhos e filhas também é ressaltado como um dos pilares dos comportamentos mais agressivos em resposta a isso pelos filhos, com atitudes de agressão e xingamento, enquanto as filhas tendem ao isolamento social frente a isso (Sólis-Câmera, Medina \& Diaz, 2015).

As práticas parentais são capazes de predizer problemas de comportamento das crianças quando há uma percepção desta realidade por parte dos pais ou cuidadores, o que ficou elucidado nos grupos de orientação parental relatados em alguns estudos (Biasoli-Alves, 2005; Brandburg \& Salvador, 2006; Neufeld, Godoi \& Rebesse, 2018).

O comportamento empático é importante para a emissão de qualquer prática parental, pois ajuda a compreender as atitudes da criança e mostra aos pais que o modo como eles interagem entre si corroboram os comportamentos apresentados no dia a dia da família (Piotrowska et al., 2016; Weber et al., 2006). Monitorar os comportamentos dos filhos, verificar se cumpriram com o que foi combinado e se estão fazendo as atividades propostas corretamente fornece oportunidade para os pais modelarem o comportamento dos filhos, seja reforçando, apresentando consequências aos comportamentos inadequados ou mostrando um modelo positivo, através de seus exemplos (Weber et al., 2006). Atitudes como envolvimento e atenção 
redobrada aos filhos e mudança de atitudes requerem conhecimento e aceitação de seus limites e de quem os cerca. $\mathrm{O}$ despreparo dos pais requer treinamento de habilidades sociais, aprendizado de competências e manejo de conflitos, temas em geral trabalhados nos Programas de intervenção (Axford et al., 2018; Neulfeuld et al., 2018).

O aprendizado social é ressaltado quando se depara com realidades de vulnerabilidade social, havendo a estruturação dos Programas como balizadora das técnicas aplicadas que possam mudar práticas disciplinares severas que desencadeiam comportamentos disfuncionais nos filhos. O desenvolvimento de competências sociais resultará em conexões seguras que expressarão sentimentos adequados. Para isso, é necessário observar o contexto cultural das famílias e orientá-las de forma positiva (Axford et al., 2018; Bagner et al., 2016; 2018; Mendes \& Biasoli-Alves, 2005; Oliveira, Gurtal \& Reis, 2018).

Na relação entre práticas e estilos parentais, destaca-se a importância da entrevista inicial na construção dos grupos de orientação parental, balizando os temas que seriam trabalhados em grupo. Assim, usar as entrevistas iniciais como forma de identificar as queixas, as situações que as originam e as percepções da mãe a respeito da criança, de suas práticas educativas e possíveis alterações que deseja mostrou ser eficaz dentro deste contexto (Pardo et al., 2015). Dentro desta premissa, a entrevista inicial age como fator de mudança, visto que leva à reflexão de experiências enquanto mãe e educadora. As questões propostas na entrevista inicial visam ampliar o olhar da mãe, direcionando a atenção para aspectos dos ambientes frequentados pela criança, para outros comportamentos ou características de seus filhos e para seu próprio comportamento enquanto educadora (Boing \& Crepaldi, 2016; Coelho \& Murta, 2017; Pardo et al., 2015).

Os grupos de orientação parental também buscam atender famílias com filhos de idade entre um ano e meio e três anos, que cada vez mais solicitam atendimento psicológico para seus filhos, com queixas frequentes envolvendo a relação com os pais, sendo as mães, na maioria das vezes, que procuram auxílio (Axford et al., 2018). Para este autor, a primeira infância é fundamental na aquisição de habilidades que serão validadas pelas atitudes dos pais e a forma com que eles lidam com as suas próprias habilidades sociais. Sendo assim, os grupos para pais podem auxiliar nas práticas parentais desde a tenra idade das crianças, o que para o autor facilitará o desenvolvimento saudável das mesmas e diminuirá o estresse dos pais, tornando o ambiente familiar mais saudável.

\subsection{Temáticas e abordagens que podem ser utilizadas na orientação de pais}

Categoria que aborda os temas que são trabalhados na orientação parental (em grupo, ou individual). Ex: "trabalha com: discussão de aspectos que influenciam no comportamento da criança e no dos pais; orientações básicas de educação da criança, tais como, regras, limites, etc.; discussão do modelo cognitivo e distorções cognitivas; noções de desenvolvimento; manejo de emoções; resolução de problemas; técnicas de relaxamento; intervenção nos pensamentos disfuncionais; entre outras coisas (artigo n. 13).

A totalidade dos artigos apresenta resultados positivos das intervenções em grupo (Axford et al., 2018; Bagner et al., 2015; Boing \& Crepaldi, 2016; Coelho \& Murta, 2017; Garcia \& Grau, 2017; Kazdin et al., 2018; Marsh et al, 2020; Mendes \& Alves, 2005; Neufeld, Godoi \& Rebesse, 2018; Pardo \& Carvalho, 2011; Silva \& Borelli, 2012; Pere et al., 2016; Piotrowska et al., 2016; Oliveira, Gurtal \& Reis, 2018; Weber et al., 2006; Vázquez et al., 2020).

Os grupos de educação parental têm demonstrado respostas significativas em diversas áreas. Percebe-se, nesse sentido, que um dos benefícios da formação do grupo está relacionado às mudanças de comportamentos considerados indesejados, bem como o desenvolvimento das habilidades sociais parentais e infantis (Coelho \& Murta, 2017; Marsh et al., 2020). De acordo com Silva e Borelli (2012), os pais vêm enfrentando dificuldades em transmitir valores, limites e normas de conduta que possam favorecer a inserção social e facilitar a vida em grupo de seus filhos. Diante disso, a orientação em grupo auxilia nessa demanda, trazendo uma realidade que não é só única e exclusiva de algumas famílias (Axford et al., 2018; Bolsoni-Silva \& Borelli, 2012; Câmera, Medina \& Diaz, 2018; Garcia \& Grau, 2017). Dessa forma, faz parte de um contexto social que se 
refere às mudanças nas relações familiares ao longo do tempo e que atinge a todos, em especial aqueles que não possuem um repertório variado e nem a possibilidade de ouvirem e serem ouvidos.

No estudo de Biasoli-Alves (2005), um grupo estruturado voltado à orientação dos pais diante das necessidades do grupo, a percepção da problemática vivida através de estratégias direcionadas ao conhecimento do funcionamento familiar e temas que condizem com as angústias e desafios educacionais oferece a possibilidade da troca de experiências e do melhor entendimento da dinâmica familiar.

Para Bolsoni-Silva et al. (2008) e Batista e Weber (2014), diversas intervenções têm sido feitas para auxiliar os pais na obtenção de um melhor relacionamento com seus filhos, entre elas a abordagem cognitiva-comportamental, que pressupõe a interação entre pensamento, emoção e comportamento interligados e a forma como se age no mundo. Esta abordagem fundamenta a intervenção grupal do Programa de Orientação de Pais, sendo um dos principais diferenciais em relação aos programas existentes na literatura, cuja base teórica é primordialmente comportamental (Neufeld, Godoi \& Rebesse, 2018).

Todas as discussões realizadas dentro de um grupo de orientação parental têm como finalidade a reflexão sobre seus próprios comportamentos, além de se auto avaliarem quanto aos seus sentimentos negativos para não magoarem os seus filhos. No estudo de Pardo et al. (2015), os autores destacaram que as mudanças devem vir dos dois lados, buscando sempre uma relação familiar funcional e saudável.

\subsection{Principais evidências de benefícios da orientação parental}

Nesta categoria, foram incluídos os dados dos estudos que demonstram as evidências de benefícios encontradas nas intervenções com orientação parental. Ex.: "é verificada uma mudança nas percepções dos pais sobre o comportamento e atitudes do filho, cuja fala passa a conter as informações dadas sobre o desenvolvimento social e afetivo nas primeiras etapas da vida e as tarefas de socialização" (artigo n. 1).

Os primeiros a perceberem modificações comportamentais em todos os cenários possíveis, como no desenvolvimento escolar, familiar e até nos cuidados da própria saúde são os pais (Marsh et al., 2020). Durante e após o fim dos grupos de orientação parental, diversos autores perceberam benefícios na relação familiar e interpessoal, além do aumento do repertório de resolução de problemas familiares. As práticas parentais tendem a ser mais saudáveis, além da existência do diálogo e trocas familiares. Assim, algumas crianças tendem a desenvolver capacidade de lidar com agentes estressores (Coelho \& Murta, 2017; Pardo et al., 2015; Oliveira, Gurtal \& Reis, 2018; Vásquez et al., 2020).

Uma pesquisa realizada por Weber et al., (2006) demonstrou mudanças significativas após a realização do grupo, tanto no que se refere aos comportamentos dos filhos quanto ao que se refere às práticas dos pais. Uma das modificações mais importantes refere-se ao conceito de amor, pois a família começou a demonstrar mais afeto na forma de abraços, palavras doces e carinho. Além disso, relataram prestar mais atenção ao que os filhos gostam, para incentivá-los a novas práticas, pois aprenderam a dar espaço e respeitar a autonomia das crianças, além de ter disponibilidade de tempo para conversas agradáveis e trocas. O autocontrole também foi citado; assim, quando os pais estão nervosos, antes de agirem, se afastam e refletem o porquê de o filho ter se comportado de tal maneira (Kazdin et al., 2018; Marsh et al., 2020; Piotrowska et al., 2016; Vásquez et al., 2020)

Boing e Crepaldi (2016) ressaltaram ainda o papel dos pais juntos na educação dos filhos e o quanto esta educação, quando fica a cargo somente da mãe, tem um profundo significado na construção dos valores que permeiam as construções afetivas da criança, visto que o pai ainda apresenta como estereótipo o papel de provedor da casa, em detrimento ao papel da mãe de mantenedora do lar e da educação dos filhos. 
A orientação de pais em grupo também objetiva saber quais agentes estressores podem estar reforçando o comportamento desadaptativo da criança, visto que esta é uma queixa recorrente na busca dos pais por um melhor entendimento e modos de atuar na educação de seus filhos (Vásquez et al., 2020).

\section{Discussão}

Esta revisão integrativa objetivou encontrar evidências de validade de intervenções em grupos voltados à orientação parental de pais de crianças. Primeiramente, destaca-se a predominância de estudos com níveis de evidência baixos em função da carência de produções científicas com metodologias mais consistentes, que possam realmente ser relevantes para uma psicologia baseada em evidência (Neufeld, Godoi \& Rebesse, 2018; Oliveira, Gurtal \& Reis, 2018; Weber et al., 2006).

As relações estabelecidas com a família na qual se nasce são as mais importantes da vida e vão representar a base do comportamento social no futuro. Para Groismann (2000), desde a infância as experiências vividas com as figuras significativas do mundo familiar vão sendo gravadas no indivíduo. Assim,os exemplos dados pelos pais em relação às suas práticas e como eles interpretam as situações, com paciência ou com violência, ficarão registrados no imaginário da criança, que desenvolverá suas ações balizadas nestas experiências(Azambuja, 2019).

A importância da intervenção na primeira infância e a utilização de programas de treinamentos para pais possibilitam uma interação positiva entre pais e filhos (Guisso, Bolze, \& Vieira, 2019). Portanto, quanto mais cedo se estabelecem as relações afetivas, mais cedo a criança desenvolverá habilidades para desenvolver-se e conviver em grupo. $\mathrm{O}$ afeto, a atenção e o tempo disponibilizado para atender às necessidades da criança favorecem as relações entre pais e filhos, pois a criança, sentindo-se segura e amada, responde assertivamente (Silva et al., 2020).

Analisando os artigos, percebe-se que em todos os documentos os pesquisadores sugerem que as práticas parentais positivas podem ser aprendidas e realizadas, a fim de manter e proporcionar o desenvolvimento saudável dos filhos, o que também foi evidenciado na revisão de literatura de Guisso, Bolze e Vieira (2019). Estes autores reforçam que a primeira infância é um marco regulador do desenvolvimento futuro da criança. Assim, treinar as habilidades dos pais na primeira infância é um prenunciador do desenvolvimento infantil.

É neste período da primeira infância que as primeiras relações interpessoais da criança se formarão e ela passará a ver o mundo e interagir com ele de forma diferente. Dependendo de suas vivências e de seu temperamento, poderão prevalecer sentimentos saudáveis em seu desenvolvimento. Para isso, os pais são fundamentais a partir do momento que conseguem enxergar seu filho como um ser independente, mas em construção e que este processo começa, ao nascer (Nelsen, 2015).

O papel dos pais foi considerado vital para o adequado desenvolvimento dos filhos na perspectiva denominada abordagem ecológica do desenvolvimento. Para Bronfenbrenner (1996), essa abordagem privilegia os aspectos saudáveis do desenvolvimento, os estudos realizados em ambientes naturais e a análise da participação do indivíduo focalizada no maior número possível de ambientes e em contato com diferentes pessoas.

Dentro desta perspectiva, Schmidt, Staudt e Wagner (2016) sugerem que a saúde familiar é incontestável frente à saúde dos filhos. Nela, se reflete todo o cuidado, todo o sentimento de pertencimento, sempre cuidando de suas necessidades básicas de manutenção. Ao viver em um país em desenvolvimento, onde o nível de pobreza e as faltas de investimentos andam entrelaçados, é inevitável que os núcleos familiares sejam afetados. A violência faz parte dessas realidades, pois a carência de educação permeia as relações entre pais e filhos. Os pais que melhor entendem essas dinâmicas são aqueles que conseguem ter empatia frente às manifestações de raiva dos filhos. Em Oliveira, Gurtal e Reis (2008), na percepção das crianças, os eliciadores da raiva mais frequentes estão relacionados à agressão verbal ou física, provenientes, na maioria das vezes, dos pais, que reagem com castigos a esta problemática (Santini \& Willians, 2016). Percebe-se aí a falta de diálogo e de conhecimento 
das necessidades dos filhos, o que pode estar atrelada a experiências negativas vividas pelos pais e/ou a manutenção de práticas parentais vivenciadas pelos mesmos.

Desta forma, o estilo e a prática educativa estão normalmente associados, uma vez que o conjunto de práticas educativas utilizadas pelos pais na interação com os filhos formará o estilo parental, ou seja, as práticas educativas que, combinadas de diferentes formas, resultam em estilos parentais diversos, de acordo com seus valores e seus padrões (Pedrosa, 2020). A relação entre as crianças e seus pais tem uma grande influência no desenvolvimento da criança, assim, potencializadas as habilidades e comportamentos parentais, os mesmos influenciam positivamente na autoestima, no sucesso escolar, no desenvolvimento cognitivo e no comportamento da criança (Pere et al., 2016).

Essa carga de atributos ao longo do tempo trouxe junto o estresse materno ao assumir muitos papéis outrora divididos com o pai, marido ou companheiro e com ele o descuido frente às atitudes dos filhos, usando, muitas vezes, a força para deter as manifestações negativas apresentadas. Esse estresse também interfere na vida conjugal dos pais, e o afeto é substituído pelas discussões e agressões que começam a fazer parte de um senso comum, distanciando cada vez mais o pai da atividade educacional de seu filho. Pensar em família no sentido que ela carrega, por ser a primeira instituição da qual se faz parte, é, antes de mais nada, se deparar com o imaginário de um lugar de afeto e segurança para a criança, pois uma visão romanceada de uma realidade bastante diferente e de relações afetivas conturbadas reflete de modo negativo na educação e criação dos filhos (Wagner, 2005).

Pardo et al. (2015) assinalam que a iniciativa de desenvolver um projeto de Grupos de Orientação a Pais decorreu da necessidade de melhor atender famílias que buscavam, cada vez mais, atendimento psicológico para seus filhos, com queixas frequentes envolvendo a relação com os pais. Estes comportamentos eram vividos também na escola e junto a seus pares, ocasionando, muitas vezes, a evasão escolar, além de práticas de violência física.

A partir de entrevistas e técnicas comportamentais, pode-se conhecer melhor a realidade familiar e, a partir dela, propor atividades de reeducação parental. A identificação dos comportamentos parentais como influência importante nos problemas de comportamento das crianças é um fator que guia o desenvolvimento dos programas de treinamento de pais (Reyno \& Mcgrath, 2006).

Educar sem usar de violência pode ser tarefa difícil, principalmente para quem teve um modelo de educação conflituosa e tem que enfrentar problemas de comportamento de seus filhos, como desobediência, mentira e agressividade. Para evitar a repetição de padrões educativos hostis, existem técnicas de educação pacíficas que podem ser ensinadas, como o treinamento de pais (Pinheiro et al., 2006). Não se pode esquecer dos diversos contextos dos quais a família faz parte e das diferenças socioeconômicas que, muitas vezes, são responsáveis pelos repertórios fracos de respostas dos pais às dificuldades comportamentais dos filhos.

Em Solís-Câmara, Medina e Diáz (2015) e Schmidt, Staudt e Wagner (2016), a terapia em grupo foi vista de forma muito positiva, pois juntos a outros pais, em especial, as mães que mais participaram dos grupos de apoio, dividiam suas angústias e expectativas, além dos grupos torná-las mais tranquilas e seguras com relação aos procedimentos educativos que estavam usando na busca do bem-estar de seus filhos.

Esta preocupação das mães é cultural frente aos papéis desempenhados pelas mulheres ao longo do tempo e o aumento das responsabilidades passa também pela formação familiar que diz que o cuidado com os filhos é papel exclusivo da mulher, cabendo a ela buscar meios de resolver os impasses de relacionamento. Neufeld et al. (2018) definem que, em situações de demanda afetiva, como a capacidade de apreender sentimentos e de identificar-se com a perspectiva do outro, manifestam-se reações que expressam essa compreensão e sentimento. 


\section{Considerações Finais}

Diante dos resultados, a literatura elencada direciona para a redução das práticas parentais disfuncionais por meio de grupos de apoio, nos quais há o treinamento de pais através de técnicas direcionadas para este fim. Percebe-se, também, que há uma escassez de ensaios clínicos e muita variabilidade de metodologia empregada nos estudos, em especial na faixa etária que compreende o nascimento aos cinco anos de idade.

Nos artigos citados, percebe-se uma grande preocupação com a violência parental, física ou psicológica, sendo possível observar que a complexidade das relações entre os pais e os seus filhos atinge a esfera familiar e individual, podendo contribuir ou prevenir a ocorrência de comportamentos disfuncionais, interferir no desenvolvimento ou agravamento de sintomas. A atuação dos grupos propicia também um momento de descarga emocional, libertando, muitas vezes, os pais do stress que causa a educação dos filhos, pois os mesmos não foram orientados para esta prática.

Os comportamentos adequados ou não das crianças são produtos da aprendizagem, ocorridos a partir das interações sociais que vivenciam, sendo os pais os primeiros exemplos a serem seguidos e, muitas vezes, o que é ensinado aos filhos foi aprendido pelos pais.

A forma com que os valores e as crenças familiares são transmitidos de pais para filhos levam ao questionamento que a transgeracionalidade, ou seja, a transmissão de significados, através das gerações, é inevitável, pois faz parte da constelação familiar, mas pode ser modificada através do conhecimento de novos repertórios educacionais, aprendidos nos grupos de treinamento parental.

Assim, aponta-se a orientação de pais como uma possibilidade bastante eficaz para problemas de relacionamento entre pais e filhos. Desse modo, pode-se assegurar que este processo consiste, atualmente, em uma alternativa para mudanças na educação parental, já que pais poderão ser adequadamente orientados e incentivados a estabelecer relações saudáveis com seus filhos. Por fim, sugere-se que sejam realizados mais estudos nesta área bem como um maior investimento em grupos, em especial no período pré-natal, pois por ser um momento de grande sensibilidade e incertezas também é um momento em que as mães estão expostas ao novo, necessitando de mais cuidados, apoio e informações, que favoreçam um melhor entendimento sobre o desenvolvimento de seus filhos.

\section{Referências}

Amorus- Marti, P. B., Byrne, S., Mateos-Inchaurrondo, A., Vaquero-Tió, E., \& Mundet-Bolós, A The implementation and evaluation of a family support program. Journals e Books, 25(2),87-93.

Asbahr, F., \& Ito. L. M. (2008). Técnicas cognitivo-comportamentais na infância e adolescência. In: Cordioli, A. V. (Org.). Psicoterapias: abordagens atuais. ( $3^{\text {a }}$ ed.): Artmed, 89-95.

Axford, N., Warner, G., Hobbs, T., Heilmann, S., Raja, A., Berry, V., Ukoumunne, O. C., Matthews, J. C., Eames, T., Kallitsoglou, A., Blower, S., Wilkinson, T., Timmons, L., \& Bjornstad, B. The effectiveness of the Inspiring Futures parenting programme in improving behavioural and emotional outcomes in primary school children with behavioural or emotional difficulties: study protocol for a randomised controlled trial. BMC Psychol, 20(6),1-3. 10.1186/s40359018-0214-7.

Azambuja, R. M. da M. (2019). Como a família lida com a dificuldade da criança diante do baixo rendimento escolar?. Research, Society and Development, 8(5), e1385702. https://doi.org/10.33448/rsd-v8i5.702

Bagner, D. M., Coxe, S., Humgerford, G. M., Garcia, D., Barroso, N. E., Hernandez, J., \& Rosa-Olivares, J. (2016). Behavioral parent training in infancy: a window of opportunity for high-risk families. J Abnorm Child Psychol, 44(5), 901-912. 10.1007/s10802-015-0089-5.

Bardin, L. (2011). Análise de conteúdo: Edições70.

Batista, A. P., \& Weber, L. N. D. (2014) Análise de programas direcionados a comportamentos exteriorizados na infância e adolescência. Psicologia Argumento, 32(78), 157-168. 10.7213/psicol.argum.32.078.

Biasoli- Alves, M. (2005). Orientação de pais: partilhar conhecimentos sobre desenvolvimento e práticas de educação como estratégia de intervenção. Texto Contexto Enferm, 14, 64-70. http://dx.doi.org/10.1590/S0104-07072005000500008. 
Boing, C., \& Crepaldi, M. A. (2016). Relação pais e filhos: compreendendo o interjogo das relações parentais e coparentais. Educar em Revista, 59(1), 17-33. https://www.scielo.br/scielo.php?pid=S0104-40602016000100017\&script=sci_abstract\&tlng=pt

Bolsoni-Silva, A. T., \& Borelli, L. M. (2012) Treinamento de habilidades sociais educativas parentais: comparação de procedimentos a partir do tempo de intervenção. Estud. pesquis. psicol.,12(1), 36-58, http://pepsic.bvsalud.org/scielo.php?script=sci_arttext\&pid=S1808-42812012000100003\&lng=pt\&nrm=i.

Bolsoni-Silva, A. T., Carrara, M., \& Marturano, M. (2008). Intervenção em grupo para pais: o que atentar quanto à intervenção e habilidades terapêuticas. In: Cavalcanti, R. (Org.). Análise do comportamento: avaliação e intervenção (pp. 102-135): Roca.

Bronfenbrenner, U. (1996) A ecologia do desenvolvimento humano: experimentos naturais e planejados: Artes Médicas.

Caballo, V. E., \& Simon, M. A. (2004). Manual de psicologia clínica infantil e do adolescente: transtornos específicos: Livraria e Editora Santos, 2004.

Caminha M. G. (2011). Treinamento de pais: aplicações clínicas. In: Caminha, M. G., \& Caminha, R. M. (Orgs.), Intervenções e treinamento de pais na clínica infantil (pp. 89-119): Sinopsys Editora

Coelho, M. V., \& Murta, C. S. (2007). Treinamento de pais: um relato de experiência. Estudos de Psicologia, 24(3),333-341.

Fundação Oswaldo Cruz (Fiocruz). (2020). Saúde mental e atenção psicossocial na pandemia Covid-19. https://portal.fiocruz.br/documento/saude-mental-eatencao-psicossocial-na-pandemia-covid-19.

Garcia, Y. A., \& Grau, I A. (2017) Entrenamiento conductual y atención plena sobre el estrés parental y relación padre-hijo. Psic.: Teor. e Pesq., 33(e3323), 19. http://dx.doi.org/10.1590/0102.3772e3323.

Gomide, P. I. C. (2001). Efeito das práticas educativas no desenvolvimento do comportamento anti-social. In: Marinho, M.L. \& Caballo, V. E. (Orgs.). Psicologia clínica e da saúde. (pp. 33-54). Londrina: UEL.

Gomide, P. I. C. (2004). Pais presentes, pais ausentes. (2ª. ed.): Vozes.

Groismann, M. (2000) Família é Deus: descubra como a família define quem você é. Rio de Janeiro; Eldorado: Núcleo-Pesquisa.

Guisso, L., Bolze, A. D. S., \& Vieira, M. L. (2019). Práticas parentais positivas e programas de treinamento parental: uma revisão sistemática da literatu ra. Contextos Clínic [online], 12(1), 226-255. https://doi.org/10.4013/ctc.2019.121.10

Kazdim, A. E., Glick, A., Pope, J., Kaptchuk, T. J., Lecaz, B., Carruba, E., McWhinney, E., \& Hamilton, N. (2018). Parent management training for conduct problems in children: enhancing treatment to improve therapeutic change. International journal of clinical and health psychology (IJCHP) 18,(2), 91-101. 10.1016/j.ijchp.2017.12.002

Lubi, A. P. L. (2003). Estilo parental e comportamento socialmente habilidoso da criança com pares. In: Brandão, M. Z. et al. (Orgs.). Sobre comportamento e cognição: a história, os avanços, a seleção por consequência em ação. (pp 536-541): Esetec.

Marsh, S., Taylor, R., Galland, B., Gerritson, S., Parag, V., \& Maddison, R. (2017). Results of the 3 Pillars Study (3PS), a relationship-based programme targeting parent-child interactions, healthy lifestyle behaviours, and the home environment in parents of preschool-aged children: A pilot randomised controlled trial. PLoS One., 17(9). 10.1371/journal.pone.0238977.

Mcmahon, R. (2007). Treinamento de pais. In: Caballo, V. E. Manual de técnicas de terapia e modificação do comportamento. (pp. 399-422): Livraria Santos Editora.

Mendes, K. D. Silveira, R. C. C. P., \& Galvão, C. M. (2008). Revisão integrativa: método de pesquisa para a incorporação de evidências na saúde e na enfermagem. Texto Contexto - Enferm., 17(4),758-64. https://doi.org/10.1590/S0104-07072008000400018

Nelsen, J.(2015). Disciplina positiva: Manole.

Pedrosa, G. F. S., \& Dietz, K. G. (2020). Representações sociais de pais/responsáveis sobre o ensino à distância no contexto da pandemia do COVID-19. Research, Society and Development, 9(8), e88984921. https://doi.org/10.33448/rsd-v9i8.4921

Silva, A. C. P. da., Danzmann, P. S., Cassel, P. A., Reginatto, M. V., \& Abaid, J. L. W. (2020). Aspectos transgeracionais, estilos parentais e maternidade: uma intervenção em grupo para a educação parental. Research, Society and Development, 9(7), e255973805. https://doi.org/10.33448/rsd-v9i7.3805

Válquez, N., et al. (2020). Social factors associated with the effectiveness of a spanish parent training program-an opportunity to reduce health inequality gap in families. International journal of environmental research and public health, 17(7). 2412. 2422. 10.3390/ijerph17072412. 